Im „Journal Club" werden Originalarbeiten aus der internationalen Fachliteratur referiert und kommentiert.

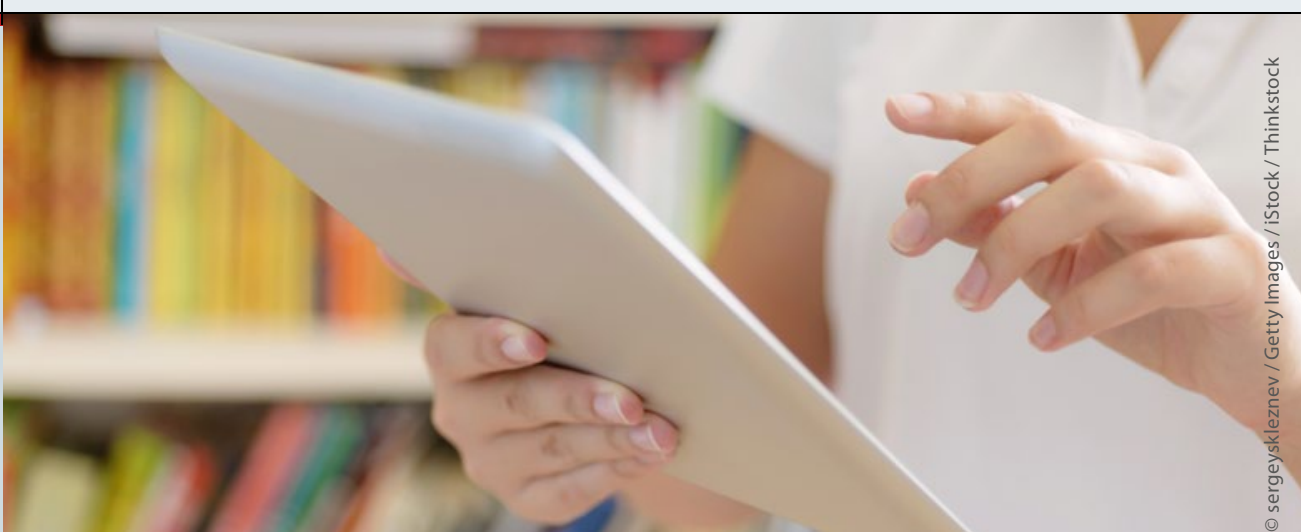

\section{Zwischen Häuslichkeit und Kurzzeitpflege}

Bedingt durch den demografischen Wandel steigt auch die Anzahl der an Demenz Erkrankten in Deutschland. Die Prognosen sind drastisch. Als Möglichkeit zur Entlastung versorgender Angehöriger wird die Kurzzeitpflege an Bedeutung gewinnen. Bereits jetzt zeichnet sich eine Zunahme der Übergänge zwischen den Settings Häuslichkeit und Kurzzeitpflege ab. Eine Delphi-Befragung nahm nun die Versorgungskontinuität von Menschen mit Demenz unter die Lupe.

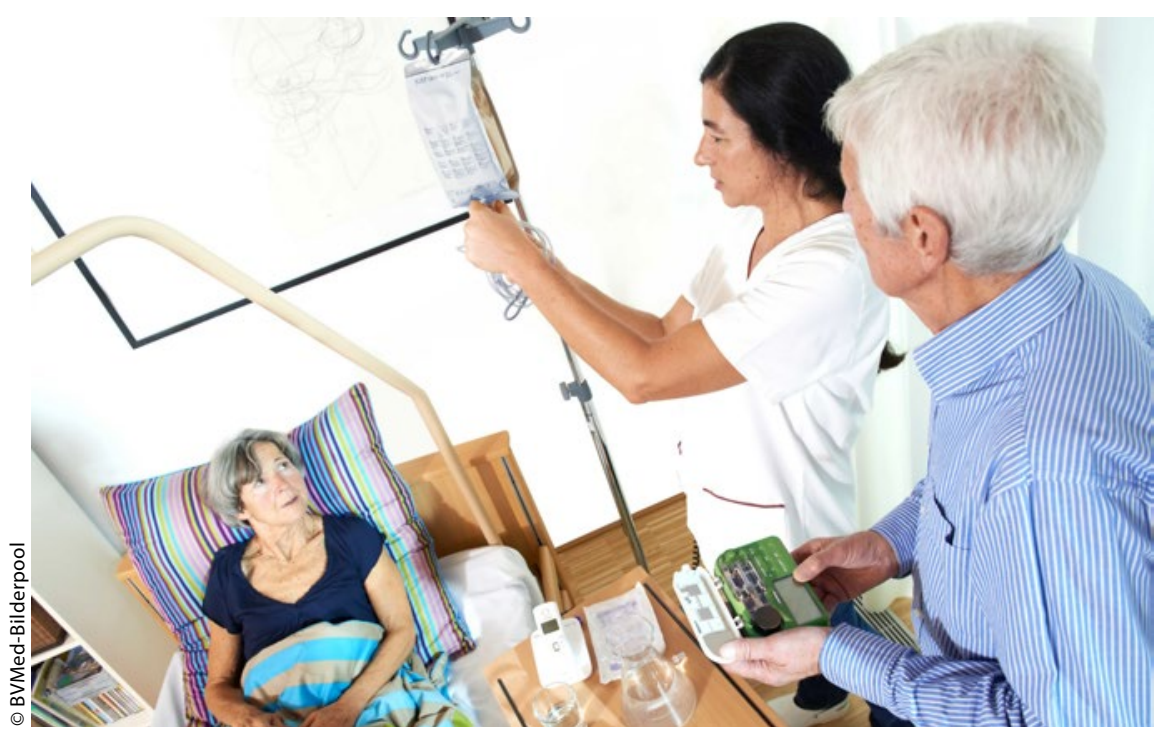

N it dem Gesetz zur Stärkung der P Pflege in Deutschland 2015 wurden die Versorgungsmöglichkeiten von Menschen mit Demenz angepasst und erweitert. Vor allem die bedarfsgerechte Pflege und Betreuung von Betroffenen in der Häuslichkeit rückt in den öffentlichen Fokus. Damit kommt Angeboten wie der Kurzzeitpflege eine höhere Bedeutung zu: Menschen mit erheblich eingeschränkter Alltagskompetenz aufgrund kognitiver Beeinträchtigungen können sie mit Pflegestufe 0 erstmals in Anspruch nehmen.
Kurzzeitpflege ermöglicht eine vorübergehende stationäre Pflege bei Verschlechterung des Gesundheitszustandes oder anderen Krisenzuständen sowie eine zeitlich begrenzte Entlastung der pflegenden Angehörigen. Bereits jetzt zeichnet sich eine Zunahme der Übergänge zwischen den Settings Häuslichkeit und Kurzzeitpflege ab. Um aber eine bestmögliche Versorgungskontinuität gewährleisten zu können, sind spezifische Kriterien für die Qualitätssicherung der Überleitung notwendig. Die vorliegende Studie wählte daher aus einer
Vielzahl bereits identifizierter Kriterien jene aus, die für eine gute Versorgungsqualität und Sicherheit von Menschen mit Demenz in Überleitungssettings besonders von Bedeutung sind. Dazu wurde eine klassische Delphi-Befragung mit einem offenen, d.h. nicht anonymen Experten-basierten Gruppen-Delphi-Design kombiniert. Des Weiteren wurden bestehende Qualitätskriterien aus Literatur und Empirie gesichtet und bewertet. Insgesamt konnten 28 Kernkriterien mit hoher Konsensstärke, wie definierte Verantwortlichkeiten, Standardisierung oder Zugänglichkeit, selektiert werden. Die Rolle pflegender Angehöriger von Menschen mit dementiellen Erkrankungen wird aufgrund ihrer Einzelfallkompetenz als zentral erachtet; ebenfalls sollte ihr individueller Unterstützungsbedarf im Vorfeld einer Überleitung berücksichtigt werden.

Gute Netzwerkarbeit, das Medikamentenmanagement sowie ein rechtzeitiger und vollständiger Informationsaustausch haben hohe Relevanz für das Gelingen von guter Überleitung. Dafür sei es förderlich, einrichtungsübergreifende Kooperationsstandards zu nutzen mit konkreten Regelungen von Verantwortlichkeiten, Kompetenzen, Aufgaben und Kommunikationsstrukturen. Um eine nachhaltig qualitativ hochwertige Überleitung zwischen Häuslichkeit und Kurzzeitpflege zu gewährleisten, empfiehlt die Forschergruppe, die identifizierten Kriterien und entsprechenden Verfahrensregelungen im Qualitäts- und Risikomanagement von Pflegeeinrichtungen zu verankern.

(dd)

S. Kuske, M. Roes, S. Bartholomeyczik. Überleitung zwischen Häuslichkeit und Kurzzeitpflege. Z Gerontol Geriat (DOI 10.1007/ s00391-015-0907-y) 